\title{
Vocabulário expressivo e variáveis regionais em uma amostra de escolares de Maceió
}

\section{Expressive vocabulary and analyze the variables in a regional sample of students in Maceió}

\author{
Vanessa Porangaba de Medeiros', Renata Kiara Lins Valença', João Alfredo Tenório Lins Guimarães², Ranilde \\ Cristiane Cavalcante Costa
}

\begin{abstract}
RESUMO
Objetivo: Caracterizar o vocabulário expressivo e analisar as variáveis regionais em uma amostra de escolares do primeiro ano do ensino fundamental de Maceió. Métodos: Participaram 72 escolares de três escolas de Maceió. Para selecionar a amostra, os escolares foram submetidos às seguintes avaliações: avaliação da fala, por meio da prova de Fonologia do ABFW; avaliação do vocabulário expressivo, por meio da aplicação da Prova de Vocabulário do ABFW e avaliação do desenvolvimento infantil, por meio da Escala de Desenvolvimento Infantil Denver. Foram realizadas análises estatística descritiva e analítica $(p<0,05)$. Resultados: Não houve diferença de desempenho lexical entre os gêneros, nem entre as faixas etárias, mas foi verificada diferença entre as escolas. Os escolares apresentaram desempenho abaixo do esperado nos campos semânticos locais, vestuário, alimentos e formas e cores. Melhor desempenho foi observado para os campos semânticos animais, meios de transporte e móveis e utensílios. Os processos de substituição mais realizados foram: substituição por co-hipônimo, por valorização do estímulo visual, por vocábulos que designam seus atributos semânticos, por hiperônimos e parassinônimos. Foram observadas diferentes designações para os vocábulos balança, escorregador, privada e casinha. Conclusão: Os escolares de Maceió que compuseram a amostra apresentaram desempenho lexical semelhante, independente do gênero e da faixa etária, mas houve diferença para a variável escola.
\end{abstract}

Descritores: Vocabulário; Testes de linguagem; Criança; Linguagem; Desenvolvimento da linguagem

\begin{abstract}
Purpose: To characterize the expressive vocabulary and analyze the variables in a regional sample of students of first grade of elementary school in Maceió. Methods: The sample was composed by 72 children from three Maceió public schools. To select the sample, the children underwent speech evaluation through the ABFW Test - Phonology; and expressive vocabulary through the ABFW - Vocabulary Test. Following, statistical analysis was conducted using Kruskal-Wallis, Mann-Whitney and Wilcoxon tests. Statistical significance was considered as $\mathrm{p}$ value smaller than 0.05. Results: There was no difference between gender and among age ranges in lexical performance. Differences among schools were observed. When compared to data from the ABFW, the children from Maceió underachieved the expected scores in the semantic fields sites, clothing, food, and shapes and colors. The best performance was observed for the fields animal, vehicles, furniture and utensils. The most recurrent substitution processes were the co-hyponym, valorization of the visual stimulus, words that designate their semantic attributes, hypernym, parasynonym or equivalents. Conclusion: The children of Maceió present a similar lexical development, independent of gender or age group, with a difference only among the schools tested.
\end{abstract}

Keywords: Vocabulary; Language tests; Child; Language; Language development

(1) Discente do Curso de Fonoaudiologia, Universidade Estadual de Ciências da Saúde de Alagoas - UNCISAL - Maceió (AL), Brasil.

(2) Curso de Fonoaudiologia, Universidade Estadual de Ciências da Saúde de Alagoas - UNCISAL - Maceió (AL), Brasil.

Conflito de interesses: Não

Contribuição dos autores: $V P M$ e $R K L V$ realizaram busca à literatura, coleta de dados, análise e interpretação dos dados e redação do manuscrito; JATLG e $R C C C$ orientaram a pesquisa.

Endereço para correspondência: Vanessa Porangaba de Medeiros. R. B, n 07, Conjunto Carajás I, Quadra A, Serraria, Maceió (AL), Brasil, CEP: 57046-390. E-mail: vanessaporangaba@ hotmail.com

Recebido em: 10/9/2012; Aceito em: 26/06/2013 


\section{INTRODUÇÃO}

A aquisição de palavras é um processo individual e heterogêneo, que pode ser influenciado por habilidades cognitivas e sociopragmáticas ${ }^{(1)}$ e marca o início da possibilidade de comunicação oral efetiva entre a criança em desenvolvimento e o mundo que a cerca ${ }^{(2,3)}$.

As primeiras palavras são adquiridas em torno de um ano; aos dois anos de idade, as crianças começam a agrupar novos vocábulos de forma rápida, caracterizando um fenômeno denominado explosão do vocabulário ${ }^{(4)}$ Quando a criança atinge os cinco anos de idade, consegue adquirir um grande número de palavras, tornando o seu vocabulário numericamente semelhante ao de um adulto. Entretanto, não é possível dizer que o vocabulário de ambos seja igual, pois a criança é capaz de selecionar características próprias na escolha das palavras que irá utilizar ${ }^{(5)}$.

O léxico pode ser visto como um compêndio de todas as palavras de uma língua ${ }^{(6)}$. Durante o processo de aquisição lexical, as crianças tendem a realizar desvios semânticos. Esses desvios ocorrem quando há a falta de correspondência entre o significado da palavra na linguagem adulta e o significado dessa mesma palavra na linguagem da criança ${ }^{(7)}$. Os desvios semânticos, também denominados de processos de substituição, foram observados com frequência em estudos que utilizaram provas de nomeação de figuras para avaliar o vocabulário ${ }^{(8-10)}$.

As pesquisas sobre a aquisição lexical permitem, atualmente, não só a descrição mais precisa do que deve ser o desenvolvimento do vocabulário, mas também do que pode ser considerado desviante ${ }^{(2)}$. A investigação do vocabulário pode ser realizada por meio da interação dialógica, ou da aplicação de testes evocativos ${ }^{(3)}$.

Para avaliação da competência lexical, existe no Brasil a prova de vocabulário do Teste de Linguagem Infantil ABFW, que vem sendo usada em pesquisas nacionais para estudo em diferentes populações, incluindo crianças com desenvolvimento típico, transtorno fonológico, distúrbio específico de linguagem e surdas.

A análise do vocabulário expressivo mostra que existe diferença de desempenho entre os campos semânticos estudados, com pior desempenho para o campo locais ${ }^{(8,11-13)}$. Quanto aos processos de substituição, estudo indica que o processo de substituição por palavra do mesmo campo semântico, denominado co-hipônimo é o mais realizado entre as crianças ${ }^{(14)}$.

$\mathrm{O}$ aspecto semântico pode ser influenciado pelo ambiente familiar e escolar, pela idade da criança, pela escolaridade materna $^{(7,15-19)}$ e pela diversidade sociocultural ${ }^{(12,14,15,17,20-22)}$. As diferenças regionais também podem influenciar no desempenho lexical, especialmente considerando-se um país com grande extensão geográfica e cultural como o Brasil. Portanto, os testes que avaliam a linguagem devem levar em conta essas diferenças regionais brasileiras ${ }^{(10)}$, que são marcadas não só por variações de desempenho, mas também por variações na denominação de vocábulos. Dessa maneira, a realidade sociolinguística deve ser considerada na aplicação de testes de desempenho semântico nas diversas populações ${ }^{(14)}$.

Considerando a influência de aspectos linguísticos, sociais, culturais e geográficos sobre a aquisição lexical, torna-se importante a realização da presente pesquisa, que teve como objetivo caracterizar o vocabulário expressivo e analisar as variáveis regionais em uma amostra de escolares do primeiro ano do ensino fundamental de Maceió.

\section{MÉTODOS}

O protocolo desta pesquisa está baseado na legislação pertinente, Resoluções № 196/96 e 251/97, do Conselho Nacional de Saúde do Ministério da Saúde para estudos com seres humanos, tendo sido aprovado pelo Comitê de Ética em Pesquisa da Universidade Estadual de Ciências da Saúde de Alagoas (UNCISAL), sob o protocolo $\mathrm{n}^{\circ}$ 1721/12.

Para obter uma amostra de escolares do primeiro ano do ensino fundamental não foram realizados cálculos estatísticos. Baseando-se em critérios geográficos e conveniência de acesso pelos pesquisadores, foram selecionadas três escolas públicas da rede municipal de ensino fundamental de Maceió (AL). Para selecionar as escolas, de forma a obter dados de diferentes regiões, a cidade foi dividida em três grandes áreas (alta, central e baixa), inserindo-se, nesta pesquisa, uma escola de cada região, denominadas de Escola 1, Escola 2 e Escola 3, localizadas respectivamente nos bairros da Serraria, Pontal da Barra e Ponta Verde.

Em cada uma das escolas, os pesquisadores promoveram uma reunião coletiva com os pais e/ou responsáveis pelas crianças do primeiro ano do ensino fundamental, a fim de explicar os objetivos da pesquisa e obter o consentimento por meio da assinatura do Termo de Consentimento Livre e Esclarecido (Resolução MS/CNS/CNEP nº 196/96 de 10 de outubro de 1996).

Os critérios de inclusão estabelecidos para esta pesquisa foram: estar regulamente matriculado no primeiro ano do ensino fundamental, ter idade entre 6 anos e 7 anos e 11 meses, e possuir autorização dos pais e/ou responsáveis para participarem da pesquisa. Foram excluídos deste estudo escolares com alteração de fala, atraso no desenvolvimento, ou sinais de perda auditiva. Os escolares foram submetidos à avaliação da fala, do desenvolvimento infantil e do desempenho auditivo durante a conversação.

Para avaliação da fala foi utilizada a Prova de Fonologia do Teste de Linguagem Infantil - $\mathrm{ABFW}^{(23)}$, composta pela nomeação de 34 figuras e imitação de 39 vocábulos, tendo sido analisada a presença de processos fonológicos produtivos, caracterizando transtorno fonológico. Para avaliação do desenvolvimento infantil foi aplicada a Escala de Desenvolvimento Infantil Denver, que abrange uma triagem em quatro áreas distintas do desenvolvimento: motricidade ampla, motricidade 
fina-adaptativa, comportamento pessoal-social e linguagem. Foi considerado atraso no desenvolvimento quando um setor apresentou dois ou mais $\operatorname{erros}^{(24)}$. O desempenho auditivo durante a conversação foi avaliado por meio da atividade de interação espontânea e pela realização dos testes que fizeram parte da seleção da amostra.

Participaram do estudo 72 escolares, sendo 32 do gênero masculino e 40 do gênero feminino. A distribuição ocorreu da seguinte maneira: 30 escolares da Escola 1, 15 da Escola 2 e 27 da Escola 3. Dentre as três escolas, foram excluídos da pesquisa 14 escolares com transtorno fonológico, três com atraso no desenvolvimento, 12 com idade superior a 7 anos e 11 meses e 14 que não estavam presentes durante a coleta.

Foi solicitada a presença do pai ou responsável pelos escolares excluídos da pesquisa em decorrência de atraso no desenvolvimento ou de alteração de fala. Os pesquisadores realizaram uma reunião coletiva a fim de orientá-los quanto ao uso de estratégias favoráveis para a estimulação da linguagem. Além disso, foram entregues documentos de encaminhamento para avaliação fonoaudiológica.

Com o objetivo de investigar o vocabulário expressivo, foi utilizada a Prova de Vocabulário do Teste de Linguagem Infantil - $\mathrm{ABFW}^{(5)}$. O teste é composto por nove campos semânticos distintos: vestuário, animais, alimentos, meios de transporte, móveis e utensílios, profissões, locais, formas e cores, e brinquedos e instrumentos musicais. As respostas foram analisadas considerando Designação por Vocábulo Usual (DVU), Não-Designação (ND) e Processos de Substituição (PS). Considerando-se as diferenças regionais presentes na designação de vocábulos das crianças de Maceió (AL) verificadas em estudo anterior ${ }^{(10)}$, foi necessário realizar algumas adaptações. Para o campo semântico brinquedos e instrumentos musicais admitiu-se como adequadas as nomeações escorrega, escorrega-rela e rela-rela para escorregador e balanço para balança. Da mesma forma, para o vocábulo privada, presente no campo semântico móveis e utensílios, foram aceitas as designações vaso, sanitário e vaso sanitário. Todas as nomeações acima descritas foram consideradas como DVU. Além disso, os escolares com mais de 7 anos foram analisados a partir do padrão das crianças de 6 anos, uma vez que o teste não propõe valores referência acima dessa idade.

Todas as avaliações foram realizadas individualmente, em sala reservada, na própria escola. O tempo médio para avaliar cada criança foi de 40 minutos, concluindo-se, portanto, a avaliação, em um único encontro. Os dados referentes à avaliação da fala foram gravados em câmera fotográfica digital Sony ${ }^{\circledR}$ Cyber-Shot 14.1 megapixels. Todos os dados foram analisados pelos pesquisadores, individualmente, e as dúvidas discutidas em reunião de consenso.

Para a análise dos dados foi utilizada estatística descritiva, com média e desvio-padrão. Na estatística analítica, para comparar o vocabulário expressivo, foi utilizado o teste de KruskalWallis na análise entre as escolas e o teste de Mann-Whitney para as variáveis gênero e faixa etária. A fim de comparar o desempenho dos escolares de Maceió com os valores esperados pelo ABFW, foi aplicado o teste de Wilcoxon. Foram considerados significativos valores de $\mathrm{p}$ menores que 0,05 .

\section{RESULTADOS}

Não houve diferença de desempenho do vocabulário expressivo analisado por meio das médias de DVU, ND e PS para as variáveis faixa etária e gênero, tendo sido verificada diferença em DVU e PS para a comparação entre as escolas, com melhor desempenho da Escola 3 e pior desempenho da Escola 1 (Tabela 1).

A comparação entre o desempenho esperado na prova de vocabulário do ABFW e o obtido pelos escolares de Maceió que compuseram o estudo, mostra que para DVU, os escolares de Maceió apresentaram desempenho abaixo do esperado para

Tabela 1. Comparação do desempenho dos escolares na prova de vocabulário expressivo segundo as variáveis faixa etária, gênero e escola

\begin{tabular}{|c|c|c|c|c|c|c|c|}
\hline & & \multicolumn{2}{|c|}{ DVU } & \multicolumn{2}{|c|}{ ND } & \multicolumn{2}{|c|}{ PS } \\
\hline & & Média & DP & Média & DP & Média & DP \\
\hline \multirow[t]{2}{*}{ Faixa etária } & 6 anos & 78,91 & 10,74 & 3,10 & 4,47 & 36,01 & 9,72 \\
\hline & 7 anos & 82,93 & 10,71 & 2,68 & 3,71 & 32,50 & 10,52 \\
\hline Valor de $p$ & & 0,122 & & 0,296 & & 0,145 & \\
\hline \multirow[t]{2}{*}{ Gênero } & Meninos & 80,81 & 8,39 & 2,06 & 2,22 & 35,15 & 8,02 \\
\hline & Meninas & 79,00 & 12,43 & 3,77 & 5,32 & 35,30 & 11,34 \\
\hline Valor de $p$ & & 0,914 & & 0,161 & & 0,662 & \\
\hline \multirow[t]{3}{*}{ Escola } & Escola 1 & 151,93 & 22,31 & 6,93 & 5,81 & 77,26 & 20,92 \\
\hline & Escola 2 & 161,86 & 19,42 & 8,66 & 15,63 & 65,46 & 17,32 \\
\hline & Escola 3 & 166,81 & 19,66 & 3,55 & 4,58 & 65,70 & 18,35 \\
\hline Valor de $p$ & & $0,021^{*}$ & & $0,016^{*}$ & & $0,054^{*}$ & \\
\hline
\end{tabular}

*Valores significativos $(p<0,05)$. Gênero e faixa etária foram analisados por meio do teste não paramétrico de Mann-Whitney. As diferenças entre as escolas foram medidas pelo teste não-paramétrico de Kruskal-Wallis.

Legenda: DVU = designação por vocábulo usual; ND = não designação; PS = processo de substituição; DP = desvio-padrão 
Tabela 2. Comparação entre o desempenho esperado na prova de vocabulário do ABFW e o desempenho obtido pelos escolares

\begin{tabular}{|c|c|c|c|c|c|c|c|c|c|}
\hline \multirow{2}{*}{ Campos semânticos } & \multicolumn{3}{|c|}{ DVU } & \multicolumn{3}{|c|}{ ND } & \multicolumn{3}{|c|}{ PS } \\
\hline & $E(\%)$ & $\mathrm{O}(\%)$ & Valor de $p$ & $E(\%)$ & $\mathrm{O}(\%)$ & Valor de $p$ & $E(\%)$ & $\mathrm{O}(\%)$ & Valor de $p$ \\
\hline Animais & 70 & 87 & $<0,001^{*}$ & 20 & 0 & $<0,001^{*}$ & 10 & 13 & 0,092 \\
\hline Alimentos & 90 & 73 & $<0,001^{*}$ & 5 & 7 & $0,028^{*}$ & 5 & 20 & $<0,001^{*}$ \\
\hline Vestuário & 80 & 60 & $<0,001^{*}$ & 0 & 0 & 0,157 & 20 & 40 & $<0,001^{*}$ \\
\hline Locais & 70 & 33 & $<0,001^{*}$ & 5 & 0 & $<0,001^{*}$ & 25 & 67 & $<0,001^{*}$ \\
\hline Profissões & 45 & 50 & 0,630 & 25 & 0 & $<0,001^{*}$ & 30 & 50 & $<0,001^{*}$ \\
\hline Meios de transporte & 70 & 82 & $<0,001^{*}$ & 5 & 0 & $<0,001^{*}$ & 25 & 18 & $<0,001^{*}$ \\
\hline Móveis e utensílios & 65 & 75 & $<0,001^{*}$ & 5 & 0 & $<0,001^{*}$ & 30 & 25 & $<0,001^{*}$ \\
\hline Briq./inst. musicais & 70 & 73 & 0,693 & 5 & 0 & $<0,001^{*}$ & 20 & 27 & $0,001^{*}$ \\
\hline Formas e cores & 85 & 80 & $0,004^{*}$ & 10 & 0 & $<0,001^{*}$ & 10 & 20 & $<0,001^{*}$ \\
\hline
\end{tabular}

*Valores significativos $(p<0,05)$ - Teste não paramétrico de Wilcoxon

Legenda: DVU = designação por vocábulo usual; ND = não designação; PS = processo de substituição; $\mathrm{E}=$ desempenho esperado na prova de vocabulário; O = desempenho obtido pelos escolares de Maceió; Briq./inst.= brinquedos e instrumentos

os campos semânticos locais, vestuário, alimentos e formas e cores. Em contrapartida, melhor desempenho foi observado nos campos animais, meios de transporte e móveis e utensílios (Tabela 2). Em relação à ND, observou-se tendência à designação, com índices abaixo ou iguais ao esperado em todos os campos semânticos, exceto alimentos, cujo índice de ND foi superior ao estabelecido. Os PS foram elevados, com diferença nos campos semânticos acima citados, que tiveram baixos desempenhos em DVU, assim como em profissões e brinquedos e instrumentos musicais (Tabela 2).

Os processos de substituição mais frequentemente utilizados pelos escolares de Maceió, em ordem decrescente foram: substituição por co-hipônimo, por valorização do estímulo visual, por vocábulos que designam seus atributos semânticos, por hiperônimos e por parassinônimos, ou equivalentes. Os demais processos de substituição foram menos frequentes ou não ocorreram (Tabela 3).

Foram observadas variações regionais durante a aplicação da prova de vocabulário expressivo. Constatou-se diferença na designação de alguns vocábulos para escolares residentes na cidade de Maceió, dentre as quais se destacam balança e escorregador, presentes no campo semântico brinquedos e instrumentos musicais, em que não foi observada nenhuma designação por vocábulo usual, conforme a denominação apresentada na Prova de Vocabulário do ABFW. Também foram encontradas variações para os vocábulos privada e casinha (Tabela 4).

\section{DISCUSSÃO}

Em relação à variável faixa etária, estudos ${ }^{(10,25)}$ realizados com crianças de 3 a 6 anos, observaram aumento dos escores de desempenho em função do aumento da idade. Outro estudo ${ }^{(7)}$ não verificou diferenças nas crianças com idades de 5 e 6 anos, embora tenham sido encontradas diferenças nas crianças com idades entre 3 e 5 anos.
Tabela 3. Frequência de uso dos processos de substituição do vocabulário expressivo

\begin{tabular}{lc}
\hline Processos de substituição & $\begin{array}{c}\text { Frequência } \\
\text { de uso }\end{array}$ \\
\hline Modificação da categoria gramatical & 163 \\
Substituição por hiperônimo & 250 \\
Substituição por co-hipônimo & 817 \\
Substituição por hipônimo & 63 \\
Criação de neologismo por analogia morfo-semântico- & 12 \\
sintática & \\
Criação de vocábulo foneticamente expressivo & 0 \\
Substituição por parassinônimo ou equivalente & 205 \\
Substituição por vocábulos que designam seus & 284 \\
atributos semânticos & \\
Substituição e/ou complementação de semiótica & 16 \\
verbal por não-verbal & \\
Substituição e/ou complementação de semiótica & 1 \\
verbal por gesto indicativo & \\
Substituição por paráfrases culturais & \\
Substituição por designação de funçães & 0 \\
Substituição por atributo de co-hipônimo & 142 \\
Substituição por paráfrases afetivas & 9 \\
Valorização do estímulo visual & 1 \\
Utilização de onomatopeia & 552 \\
Segmento ininteligível & 3 \\
\hline & \\
\hline
\end{tabular}

A literatura aponta que, por volta dos 5 anos de idade, o vocabulário de uma criança é numericamente semelhante ao de um adulto em seu dia-a-dia ${ }^{(5)}$. Esse fato pode justificar uma aproximação com o desempenho lexical dos escolares com idade superior a 5 anos, como os que compuseram a amostra desta pesquisa.

Quanto à variável gênero, o resultado encontrado concorda com estudos ${ }^{(10,25)}$ que não verificaram diferença entre meninos 
Tabela 4. Descrição de variações regionais encontradas na amostra de escolares

\begin{tabular}{|c|c|c|c|c|}
\hline Designação esperada & & ções regionais encontr & & \\
\hline $\begin{array}{l}\text { Casinha } \\
(n=1)\end{array}$ & $\begin{array}{c}\text { Casa } \\
(n=68)\end{array}$ & $\begin{array}{l}\text { Casinha de cachorro } \\
\qquad(\mathrm{n}=3)\end{array}$ & --------------- & --------------- \\
\hline $\begin{array}{l}\text { Escorregador } \\
(n=0)\end{array}$ & $\begin{array}{l}\text { Escorrega rela } \\
\qquad(n=35)\end{array}$ & $\begin{array}{l}\text { Rela rela } \\
\qquad(\mathrm{n}=19)\end{array}$ & $\begin{array}{l}\text { Escorrega } \\
\qquad(n=8)\end{array}$ & $\begin{array}{l}\text { Outros } \\
(n=10)\end{array}$ \\
\hline $\begin{array}{l}\text { Balança } \\
(n=0)\end{array}$ & $\begin{array}{l}\text { Balanço } \\
(n=61)\end{array}$ & $\begin{array}{l}\text { ND } \\
(n=5)\end{array}$ & $\begin{array}{c}\text { Outros } \\
(n=6)\end{array}$ & ------------- \\
\hline $\begin{array}{l}\text { Privada } \\
(\mathrm{n}=5)\end{array}$ & $\begin{array}{l}\text { Vaso, vaso sanitário e sanitário } \\
\qquad(\mathrm{n}=53)\end{array}$ & $\begin{array}{c}\text { Banheiro } \\
(n=13)\end{array}$ & $\begin{array}{l}\text { Pinico } \\
(n=4)\end{array}$ & $\begin{array}{l}\text { ND } \\
(n=2)\end{array}$ \\
\hline
\end{tabular}

e meninas. Pesquisas anteriores sugerem que a diferença para essa variável ocorre em idades mais precoces e que nas habilidades comunicativas e na organização linguística as meninas falam mais cedo e produzem mais discursos que os meninos ${ }^{(25,26)}$.

$\mathrm{Na}$ literatura consultada, não foram encontrados estudos que utilizaram como variável escolas de diferentes regiões de uma mesma cidade. A razão de haver diferença de desempenho entre as escolas públicas pode ser justificada pela diferença de poder aquisitivo da população residente nos bairros em que estão localizadas as escolas. Apesar de serem todas escolas municipais, a Escola 3 localiza-se em um bairro considerado elitizado da cidade de Maceió, diferentemente dos bairros onde estão localizadas as Escolas 1 e 2.

Essa hipótese apoia-se no fato de que os aspectos socioculturais, como baixo poder aquisitivo e baixos níveis de educação, podem interferir na aquisição do vocabulário ${ }^{(14,16,17,20,21)}$. Além disso, a acessibilidade à brinquedos e objetos culturais, bem como a realização de atividades e reuniões compartilhadas com os pais, são importantes para o desenvolvimento infantil ${ }^{(17)}$.

Portanto, deve ser considerada a realidade sociolinguística ao se aplicar testes de desempenho semântico nas diversas populações ${ }^{(14)}$ e ser discutido o grande número de variáveis sociais que influenciam no desenvolvimento da criança, a fim de que a comunidade, a escola e os serviços de saúde possam trabalhar juntos em projetos que aperfeiçoem o desenvolvimento infantil ${ }^{(20,21)}$.

O desempenho lexical obtido pelos escolares de Maceió foi comparado com os valores esperados pela prova de vocabulário do Teste de Linguagem Infantil ABFW (Tabela 2). Alguns dos campos semânticos com pior desempenho coincidiram com estudos anteriores, como locais $^{(8,11,13)}$, vestuário, alimentos ${ }^{(11)}$ e formas e cores. O campo semântico locais também foi considerado como o mais difícil em estudos com crianças com transtorno fonológico ${ }^{(27)}$ e surdas ${ }^{(28,29)}$.

$\mathrm{O}$ corpus desta pesquisa é formado por escolares sem atraso no desenvolvimento ou problemas de fala. O resultado mostra que o desempenho dos escolares de Maceió para os campos semânticos locais, vestuário, alimentos e formas e cores não coincide com o padrão esperado na prova de vocabulário do ABFW. Assim, questiona-se se as dificuldades encontradas nesses campos devem ser consideradas como desempenho inadequado, ou apenas diferentes do padrão proposto.
Pesquisa anterior destacou que os testes que têm o propósito de avaliar a competência lexical devem considerar as dimensões do Brasil e suas diversidades regionais ${ }^{(10,22)}$. Desta forma, existe a necessidade de se trabalhar pela padronização que possa abranger diferentes estados e regiões, na tentativa de obter valores que representem a média de desempenho nacional para crianças com desenvolvimento típico ${ }^{(10)}$. Embora seja destacada a importância de se considerar as diferenças regionais e a necessidade de se estabelecer padronização dos testes de linguagem, fica a reflexão sobre a possibilidade de realização efetiva dessa proposta.

A partir de instrumentos para avaliação lexical, estudo ${ }^{(3)}$ concluiu que para investigação do vocabulário infantil é preferível utilizar a metodologia de interação dialógica do que os testes evocativos e as listas de verificação. Entretanto, não se pode deixar de considerar que a objetividade presente nos testes de evocação possibilita uma análise mais fidedigna, pois os resultados são analisados a partir dos valores propostos. Além disso, os testes também são importantes para a realização de pesquisas científicas, pois permitem a reprodução do método sem dificuldade.

De um lado, a interação dialógica melhor representa a singularidade da linguagem de uma criança, de outro, os testes de nomeação trazem a objetividade da análise. A partir do paradoxo, torna-se importante refletir qual a melhor metodologia a ser utilizada na clínica e em pesquisas que investigam o vocabulário infantil.

As crianças desta pesquisa apresentaram baixos índices para ND, concordando com outros estudos que referem ser mais comum a criança realizar um processo de substituição do que não nomear um objeto ${ }^{(8-10)}$. Assim, a tendência dos escolares em nomear, definir ou caracterizar as imagens indica que apresentaram algum tipo de conhecimento sobre as figuras que compõem o teste.

Para nomeação de figuras devem ser levadas em consideração as características culturais, de desenvolvimento, familiaridade com o vocábulo, falta de clareza visual das figuras e a frequência de acessibilidade aos objetos no cotidiano dos participantes ${ }^{(11-12)}$.

Os processos de substituição mais frequentemente encontrados (Tabela 3) confirmaram pesquisa anterior: substituição 
por co-hipônimo ${ }^{(14)}$, por valorização do estímulo visual ${ }^{(14)}$, por vocábulos que designam seus atributos semânticos, por hiperônimos e por parassinônimos ou equivalentes.

O processo de substituição por co-hipônimo é denominado de desvio de superextensão em estudo que investigou o vocabulário infantil. É um processo comumente observado na aquisição lexical e ocorre quando a criança, na falta de um termo mais apropriado, busca outro vocábulo que pertence ao mesmo campo semântico ${ }^{(7)}$.

O processo de substituição por valorização do estímulo visual ocorre quando a criança nomeia um componente que se destaca na figura ${ }^{(5)}$. Esse foi o segundo processo mais utilizado pelos escolares e mostra que características específicas das imagens podem contribuir para que esse processo esteja entre os mais prevalentes realizados pelos escolares.

O processo de substituição por vocábulos que designam seus atributos semânticos foi o terceiro mais utilizado pelos escolares de Maceió. Ocorre quando a criança nomeia qualidades da imagem, sendo pertinentes ou não. Esse processo não foi descrito em outras literaturas, mas mostra que, muitas vezes, os escolares não conseguem se lembrar do vocábulo-alvo e por isso descrevem características que representam a figura.

A substituição por hiperônimo foi o quarto processo de maior ocorrência e surge quando há substituição de um vocábulo por outro semanticamente mais abrangente ${ }^{(5)}$. Os termos semanticamente mais abrangentes de um campo semântico são considerados menos marcados e mais fáceis de serem aprendidos e lembrados do que os mais marcados ${ }^{(6)}$. Isso indica que os escolares ainda realizam uma generalização, usando traços semânticos que não especificam o vocábulo.

O processo de substituição por parassinônimo é caracterizado por expressões que remetem ao mesmo significado ${ }^{(5)}$. No que se refere a esse processo, merece destaque a nomeação do vocábulo viatura, presente no campo semântico meios de transporte, uma vez que nenhum dos escolares deste estudo nomeou adequadamente essa figura. Observou-se que 37,5\% dos escolares nomearam viatura como carro de polícia e os outros $62,5 \%$ nomearam como carro ou polícia. Isso nos faz refletir sobre a possibilidade de não se tratar de uma substituição, mas de uma variação semântica. Sugere-se que carro de polícia seja aceito como DVU quando a prova de vocabulário for aplicada na cidade de Maceió.

Os vocábulos que apresentaram variações na designação foram: balança, escorregador, privada e casinha (Tabela 4). Não houve nenhuma designação usual para os vocábulos balança e escorregador. De fato, para os moradores da cidade de Maceió o vocábulo balança representa máquina que serve para aferir peso, comumente encontrada em locais que vendem produtos a quilo, tais como supermercados. $\mathrm{O}$ brinquedo que serve para balançar, frequentemente encontrado em parques infantis, é denominado balanço. Em relação ao vocábulo escorregador foi possível perceber que as designações escorrega-rela, rela-rela e escorrega foram as mais predominantes entre os escolares.
Quanto ao vocábulo privada, pôde-se perceber que, embora cinco crianças da amostra tenham realizado a nomeação esperada pelo teste, a maioria dos escolares utilizou as denominações vaso sanitário, vaso e sanitário. Todas as designações descritas para balança, escorregador e privada concordam com pesquisa anterior $^{(10)}$, que recomenda considerar as variações encontradas como designações usuais para Maceió.

Para o vocábulo casinha presente no campo semântico brinquedos e instrumentos musicais, apenas uma criança da amostra realizou a designação esperada. As demais, realizaram o processo de substituição denominado modificação da categoria gramatical, no qual o vocábulo casinha foi nomeado como casa. Pode-se supor que a figura da casinha presente na prova de vocabulário não tenha sido identificada como uma casinha de brinquedo e por essa razão, a maioria das crianças utilizou a denominação casa. Neste caso, a variação encontrada poderia ter sido influenciada pela imagem e não por uma variação regional.

As diferenças na designação de vocábulos observadas entre os escolares de Maceió traz para discussão não apenas a necessidade de padronizar os testes que avaliam desempenho lexical por meio da média nacional, mas também a importância de se considerar as variações. Salienta-se que esse objetivo só poderá ser alcançado por meio de estudos multicêntricos, com a colaboração de pesquisadores locais inseridos em diferentes regiões do Brasil.

Os testes que avaliam a linguagem são extremamente importantes e necessários, tanto para a prática clínica, quanto para a utilização em pesquisas científicas, mas os profissionais que fazem uso desses instrumentos precisam considerar as variações que podem ocorrer.

O estudo apresenta como limitação uma amostra reduzida de escolares que precisa ser ampliada e selecionada a partir de critérios estatísticos. Entretanto, já foi possível perceber que o vocabulário infantil sofre influência de fatores sociais, culturais e econômicos. Sugere-se que novos estudos sejam realizados neste âmbito, a fim de se caracterizar o vocabulário dos escolares de diferentes regiões do país.

\section{CONCLUSÃO}

Os escolares do primeiro ano do ensino fundamental de Maceió que compuseram esta amostra apresentaram desempenho lexical semelhante, independente do gênero e da faixa etária, mas houve diferença com relação à localização das escolas, o que reflete possível interferência de fatores socioeconômicos.

\section{REFERÊNCIAS}

1. Gândara JP, Befi-Lopes DM. Aquisição lexical no desenvolvimento normal e alterado de linguagem: um estudo experimental. Rev Soc Bras Fonoaudiol. 2008;13(supl.2008):1119-24.

2. Gândara JP, Befi-Lopes DM. Tendências da aquisição lexical em 
crianças em desenvolvimento normal e crianças com alterações específicas no desenvolvimento da linguagem. Rev Soc Bras Fonoaudiol. 2010;15(2):297-304.

3. Bastos JC, Ramos APF, Marques J. Estudo do vocabulário infantil: limitações das metodologias tradicionais de coleta. Rev Soc Bras Fonoaudiol. 2004;9:1-9.

4. Vidor DCGM. Aquisição lexical inicial por crianças falantes do português brasileiro: discussão do fenômeno da explosão do vocabulário e da atuação da hipótese do viés nominal [dissertação] Porto Alegre: Pontifícia Universidade Católica do Rio Grande do Sul, 2008.

5. Befi-Lopes DM. Vocabulário. In: Andrade CRF, Befi-Lopes DM, Fernandes FDM, Wertzner HF. ABFW: teste de linguagem infantil, nas áreas de fonologia vocabulário, fluência e pragmática. Carapicuíba: Pró-Fono; 2004. p. 33-49.

6. Finegan E. Language. Its structure and use. Forth Worth/ Texas: Harcourt Brace \& Company, 1994.

7. Hage SRV, Pereira MB. Desempenho de crianças com desenvolvimento típico de linguagem em prova de vocabulário expressivo. Rev CEFAC. 2006;8(4):419-28.

8. Brancalioni AR, Marini C, Cavalheiro LG, Keske-Soares M. Desempenho em Prova de vocabulário de crianças com desvio fonológico e com desenvolvimento fonológico normal. Rev CEFAC. 2011;13(3):428-36.

9. Athayde ML, Mota HB, Mezzomo CL. Vocabulário expressivo de crianças com desenvolvimento fonológico normal e desviante. Pro Fono. 2010;22(2):145-50.

10. Costa RCC. Competência lexical e metafonológica em pré-escolares com transtorno fonológico [dissertação] São Paulo: Universidade Federal de São Paulo, Escola Paulista de Medicina, 2010.

11. Kaminski TI, Mota HB, Cielo CA. Consciência fonológica e vocabulário expressivo em crianças com aquisição típica da linguagem e com desvio fonológico. Rev CEFAC. 2011;13(5):81330.

12. Miranda MC, Pompéia S, Bueno OFA. Um estudo comparativo das normas de um conjunto de 400 figuras entre crianças brasileiras e americanas. Rev Bras Psiquiatr. 2004;26(4):226-33.

13. Torres MLGM, Maia HA, Perissinoto J, Assencio-Ferreira VJ. Descrição do léxico expressivo de crianças aos 5 anos de idade. Rev CEFAC. 2002;4:241-51.

14. Carvalho LS. Variação sociolinguistica e aquisição semântica: um estudo sobre o perfil lexical pelo teste ABFW numa amostra de crianças em Salvador-BA. Rev Soc Bras Fonoaudiol. 2009;14(supl. 2009):1450-5.
15. Cachapuz RF. Halpern R. A influência das variáveis ambientais no desenvolvimento da linguagem em uma amostra de crianças. Revista da AMRIGS. 2006;50(4):292-301.

16. Rowe ML. Child-directed speech: relation to socioeconomic status, knowledge of child development and child vocabulary skill. J Child Lang. 2008;35:185-205.

17. Ferreira SHA, Barrera SD. Ambiente familiar e aprendizagem escolar em alunos da educação infantil. Psico. 2010;41(4):462-72.

18. Scopel RR, Souza VC, Lemos SMA. A influência do ambiente familiar e escolar na aquisição e no desenvolvimento da linguagem: revisão de literatura. Rev CEFAC. 2012;14(4):732-41.

19. Maria-Mengel MRS, Linhares MBM. Fatores de risco para problemas de desenvolvimento infantil. Rev Lat Am Enfermagem. 2007; $15: 837-42$.

20. Basílio CS, Puccini RF, Silva EMK, Pedromônico MRM. Living conditions and receptive vocabulary of children aged two to five years. Rev Saúde Pública. 2005;39(5):725-30.

21. Gaskell MG, Ellis AW. Word learning and lexical development across the lifespan. Philos Trans R Soc Lond B Biol Sci. 2009;364(1536):3607-15.

22. Oliveira e Britto DB, Teixeira ACA, Xavier AS. A avaliação do vocabulário valorizando o dialeto local - um olhar além dos testes padronizados. Rev Soc Bras Fonoaudiol. 2009:14(supl. 2009):2389.

23. Wertzner HF. Fonologia. In: Andrade CRF, Befi-Lopes DM, Fernandes FDM, Wertzner HF. ABFW: teste de linguagem infantil, nas áreas de fonologia vocabulário, fluência e pragmática. Carapicuíba: Pró-Fono; 2004. p. 5-31.

24. Frankenburg WK, Dodds JB. The Denver developmental screening test. J Pediatr. 1967;71(2):181-91.

25. Ferracini F, Capovilla AGS, Dias NM, Capovilla FC. Avaliação de vocabulário expressivo e receptivo na educação infantil. Rev. Psicopedag. 2006;23(17):124-33.

26. Sandri MA, Meneghetti SL, Gomes E. Perfil comunicativo de crianças entre 1 e 3 anos com desenvolvimento normal de linguagem. Rev CEFAC. 2009;11(1):34-41.

27. Mota HB, Kaminski TI, Nepomuceno MRF, Athayde ML. Alterações no vocabulário expressivo de crianças com desvio fonológico. Rev Soc Bras Fonoaudiol. 2009;14(1):41-7

28. Costa MCM, Chiari BM. Verificação do desempenho de crianças deficientes auditivas oralizadas em teste de vocabulário. Pro Fono. 2006;18(2):189-96.

29. Ferreira MIO, Dornelas SA, Teófilo MMM, Alves LM. Avaliação do vocabulário expressivo em crianças surdas usuárias da Língua Brasileira de Sinais. Rev CEFAC. 2012;14(1):9-17. 